83. Martres, M. J., Pick, M., Soru-Escaut, I., Axisa, F. $\quad$ 1972, Nature, 236, 63, 25.

84. Zaitsev, V. V. 1970, Izv. Vuzov Radiofiz., 13, 837.

85. Smith, D. F. $\quad$ 1970, Adv. Astr. Astrophys., 7, 147.

86. Smith, D. F. 1970, Solar Phys., 15, 202.

87. Smith, D. F., Fung, P. C. W. 1971, J. Plasma Phys., 5, 1, 30.

88. Smith, D. F. 1972, Solar Phys., 23, 191.

89. Melrose, D. B. 1970, Austr. J. Phys., 23, 885.

90a. Zheleznyakov, V. V., Zaitsev, V. V. 1970, Soviet Astr., 14, 1, 147.; 90b. 1970, 14, 2, 250.

91. Zheleznyakov, V. V., Mityakov, Rapoport, V. O. $\quad$ 1972, Solar Phys., 33, 123.

92. Aubier, M., Boischot, A. 1972, Astr. Astrophys., 19, 343.

93. Zaitsev, V. V., Mityakov, N. A., Rapoport, V. O. 1972 , Solar Phys., 24, 444.

94. Harvey, C., Aubier, M. 1973, Astr. Astrophys., 22, 1.

95. Smith, D. F., Pneuman, G. W. 1972, Solar Phys., 25, 461.

96. Chiu, Y. T. 1970, Solar Phys., 13, 420.

97. Kuckes, A. F., Sudan, R. N. 1971, Solar Phys., 17, 194.

98. Labrum, N. R., Stewart, R. T. 1970, Proc. Astr. Soc. Australia, 1, 7, 316.

99. Fokker, A. D. 1970, Solar Phys., 11, 92.

100. Daene, H. N., Formichev, V. V. 1971, Heinrich Hertz Institut Publ., 2, 6, 241.

101. Smith, D. F. $\quad 1970$, Solar Phys., 13, 444.

102. Stone, R. G., Fainberg, J. 1971, Solar Phys., 20, 106.

103. Dulk, G. A., Smerd, S. F. $\quad$ 1971, Austr. J. Phys., 24, 185.

104. Dulk, G. A. 1971, Austr. J. Phys., 24, 177.

105. Dulk, G. A., Altschuler, M. D., Smerd, S. F. $\quad$ 1971, Astrophys. Letters, 8, 235.

106. Aubier, M., De la Noë, J. 1971, Astr. Astrophys., 12, 491.

107. Stepanov, A. V. 1970, Radiofisika, 13, 9.

108. Smith, D. F. $\quad 1971$, Astrophys. $J$., paper I (108a) 170, 559; paper II (108b) 174, 121; paper III (108c) $174,643$.

109. Lacombe, C., Pedersen, B. M. 1971, Astr. Astrophys., 15, 406.

110. Smerd, S. F., Dulk, G. A. 1971, Solar Magnetic Fields, Ed. R. Howard, IA U Symposium 43, 616.

111. Kundu, M. R., Erickson, W. C., Jackson, P. D., Fainberg, J. 1970, Solar Phys., 14, 394.

112. Akinyan, S. T., Mogilevsky, E, I, Böhme, A., Krüger, A. 1971 , Solar Phys., $20,112$.

113. Hansen, R. T., Garcia, C. J. 1971, Proc. Astr. Soc. Australia, 2, 1, 57.

114. De Groot, T. 1970, Solar Phys. 14, 176.

115. Abrami, A. 1970, Solar Phys. 11, 104.

116. Gotwold, B. L. 1972, Solar Phys., 25, 232.

117. Rosenberg, H. 1970, Astr. Astrophys. 9, 159.

118. Abrami, A. 1972, Nature, 238, 25.

119. Rosenberg, H. 1972, Solar Phys. 25, 188.

120. Rosenberg, H., Tarnstron, G. 1972, Solar Phys., 24, 210.

121. Gnesdilov, A. A. $\quad 1970$, Astr. Z. U.S.S.R., 47, 76.

122. Bougeret, J. L. 1973 , Astr. Astrophys., in press.

123. Elgaroy $\emptyset$, Ugland, O. 1970 , Astr. Astrophys., 5, 372.

124. Trotter, E. D., Newkirk Jr. G. 1971, Solar Phys., $20,372$.

125. Fainberg, J., Stone, R. G. 1971, Astrophys. J., 164, L123.

\title{
B. CONTINUUM RADIATION FROM THE GALAXY
}

\section{F. D. Drake}

The breadth of studies of the galactic continuum radiation has greatly expanded since the last reporting period due to the discovery of new types of emitting objects, and new interest in aspects of previously known phenomena. The number of papers published has been of the order of a thousand, and it is possible here only to give a representative collection and to indicate some of the important papers.

A most important development was the discovery of radio emission from a number of stellar galactic objects of various types. These included novae $(59,62,177)$ which were seen to change their intensity and spectra in accordance with the theoretically predicted evolution of radio emission 
from an expanding shell. High resolution interferometer studies also led to the detection of normal stars $(63,66,175)$ such as Algol and Antares B, the radiation of which in some cases is variable. Several $X$-ray sources were detected $(64,65,67,176)$, among which Scorpius $X-1$ was found to have a complex structure and variable radio emission. Infra-red objects were searched for and some found $(\mathbf{7 1}, \mathbf{9 2}, \mathbf{1 9 1})$. Some notable work was done on flare stars (108) but activity in this field was relatively low. An entire issue of Nature, Review A was devoted to a remarkable outburst of the $\mathrm{X}$-ray source Cygnus X-3. A review of stellar radio emission is given in 66 .

Pulsars continued to attract a great deal of attention, with effort being devoted to defining the radio phenomenon with great precision in order to determine how particles in the spinning magnetic field of the neutron star gives rise to the complex results observed. A large number of papers on the general observational properties of pulsars appeared $(2,14,23,36,37,39,43,47,51,53$, $54,76,77,78,80,102,105,111,112,118,142,144,167,168,172,173,174)$. Spectral data were given in 76 and 77. A number of studies were made of the fine structure and systematic morphology of pulsar pulses and pulse trains $(42,79,109,110,122,137,143,156,157,158,159,161,162$, $195,196,197)$. These showed that the pulse intensities of virtually all pulsars were modulated periodically, usually with two modulation periods, one acting towards the center of the pulse and the other towards the ends $(195,196,197)$. This is related to drifting or marching subpulses. Pulsar nulling was also detected (195), and discrete transformations of mean pulse shape (196). A great deal of research was done on the Crab Nebula Pulsar $(35,52,56,113,115,117,119,120,131$, $135,140,148,150,154,157,159)$. This produced the statistics of the very strong occasional pulses of this pulsar, showed they were of duration of the order of $100 \mu \mathrm{s}$, and showed the precursor component to be highly polarized, while the other components are not. Studies of the details of pulsar polarization and Faraday rotation were made $(19,50,57,114,115,116,119,151,155,162)$. The timing of pulsar frequencies continued $(\mathbf{7 2}, \mathbf{8 1}, \mathbf{8 2}, \mathbf{1 3 0}, \mathbf{1 3 1})$, and showed all pulsars to be spinning down as expected in the rotating neutron star theories. The timing data for only the Crab Nebula pulsar produced good data on the torque mechanism in pulsars. The search for new pulsars continued, but with reduced effort $(10,16,21,22,136)$.

There were a large number of theoretical papers $(145,146,147,149,150,160,192,194)$ but the theory of the radiation mechanism remained vexed. A general review of pulsars is given in Review C (152), and there are much data on the Crab Nebula pulsar in Review B. A general tabulation of observed pulsar parameters is given in $\mathbf{1 6 8}$.

A large number of detailed maps of supernova remnants were produced, many of these containing high quality polarization information $(25,26,27,28,29,30,31,46,61,83,90,98,99$, $100,101,103,144,172,183,184,188)$. These generally showed a geometry in the magnetic field which correlated well with the structure of the remnant, but no general rule emerged. In some remnants, fields seemed primarily radial, while in others they followed the outer contours of the remnant. A catalog of 117 possible remnants was produced (31). The Crab Nebula continued to draw particular interest $(34,45,68,87,128,140,184)$. It was found that, contrary to previous reports, the radio spectrum at millimeter wavelengths contains no excess radiation, but is a continuation of the spectrum seen at lower frequencies (128). An object related to a supernova, the Gum Nebula, was recognized as the fossil Stromgren sphere associated with the supernova of Vela X $(5,11)$. This led to the development of theories concerning the morphology and evolution of such objects (12).

The study of $\mathbf{H}$ in regions made great strides, primarily through the application of high resolution pencil-beam and synthesis instruments, recombination line data, and improved and more general theories. There were a number of general surveys made of $\mathrm{H}$ iI regions $(18,24,86,123,166,185$, 187). Many very high resolution maps were produced $(48,55,84,85,87,89,128,138,141,165$, $179,180,182)$, including some made by aperture synthesis $(179,180,182)$. These showed the density distribution to be highly irregular, with very dense knots which are optically thick at rather high radio frequencies. An important development was the establishment of a class of $\mathbf{H}$ in regions called 'compact', where densities are high and there is evidence of young age and possibly active star formation $(17,73,124,125,126,139,164,169)$. Improved instrumentation permitted major 
progress in the studies of planetary nebulae, where again some evidence for dense knots was found $(74,88,127,129,163,170,181)$.

New general surveys of the galactic radio emission were made with improved sensitivity and resolution at $1.414,2.695$, and $5.0 \mathrm{GHz}(6), 1410 \mathrm{MHz}(95), 408 \mathrm{MHz}(133), 610.5 \mathrm{MHz}(178$, 186), and $2695 \mathrm{MHz}(185)$. Surveys of the distributed galactic background alone were made (60, 97, 132). There was discussion of such surveys $(39,93,94,134)$ with a general review in 94 . Few authors saw any evidence for a galactic halo. Spacecraft were used to measure the low frequency spectrum and crude distribution of galactic radiation down to $0.2 \mathrm{MHz}$, from which were derived the spectrum of low energy cosmic ray electrons $(3,15,20,49,58)$. By similar techniques, the low radio frequency free-free absorption of the interstellar medium was observed $(4, \mathbf{1 3})$. From such surveys statistics were constructed on the distribution of thermal and non-thermal sources $(7,189,190)$.

The development of much improved millimeter wave telescopes and receiving systems allowed major progress in millimeter wave flux density measurements and, in some cases, the mapping of brightness distributions $(1,32,38,44,121,128,191,193)$. These data, with those previously mentioned allowed the construction of far more extensive catalogs of radio source spectra $(5,41$, 69, 153).

Special attention was given to the monitoring of variable radio sources $(8,96,104,106)$, and it was found that some sources vary in times of days, and some, such as Cygnus X-3 in times of hours (A). Efforts continued to measure the detailed polarization of radio sources $(9,33,75)$.

As usual, a source of special interest was the galactic center source, Sagittarius A $(38,70,91)$. Observations designed to detect the presence of a black hole in the galactic nucleus gave encouraging but inconclusive results (40).

One major search for extraterrestrial intelligent radio signals was conducted at $927 \mathrm{MHz}(\mathbf{1 7 1})$. Some fifty stars were examined with negative results. This search is still in progress, and two more are being pursued in the United States.

\section{BIBLIOGRAPHY}

\section{Reviews}
A. Special Issue on Cygnus X-3, Nature Physical Science 239, No. 95, 1972.
B. IAU Symposium No. 46, The Crab Nebula, D. Reidel, 1971.
C. Pulsars, F. G. Smith, 1972, Reports on Progress in Physics, in press.

\section{Papers}

1. Akvilonova, A. V., et al. $\quad$ 1972, Astron. Journ., USSR, 49. 102.

2. Alekseev, Yu. I. 1971, Astron. Circ., 635, 1.

3. Alexander, J. K., Brown, L. W., Clark, T. A., Stone, R. G. $\quad$ 1970, Astr. Astrophys., 6, 476.

4. Alexander, J. K., Brown, L. W. 1971, Bull. Amer. Astr. Soc., 3, 469.

5. Alexander, J. K., Brandt, J. C., Maran, S. P., Stecher, T. P. 1971, Ap. J. 167, 487.

6. Altenhoff, W. J., Downes, D., Goad, L., Maxwell, A., Rinehardt, R. 1970, Astron. Astrophys. Suppl. Ser., 1, 319.

7. Altenhoff, W. J., Wilson, T. L. $\quad$ 1970, Nature, 225, 245.

8. Benediktov, E. A., Vuzov Izvestia 1970, Radiofizika, 13, 1474.

9. Bologna, J. M., McClain, E. F., Sloanaker, R. M. $\quad$ 1969, Astrophys. J., 156, 815.

10. Bourgois, G., Guelin, M., Rieu, N. Q. 1969, Nature, 222, 963.

11. Brandt, J. C., Stecher, T. P., Crawford, D. L., Maran, S. P. 1971, Ap. J., 163, L99.

12. Brandt, J. C., Maran, S. P. 1972 , Nature, 235, 38.

13. Brezgunov, V. N., Daggesamanskiy, R. D., Udaltsov, V. A. $\quad$ 1972, Astrophys. J., 9, 117.

14. Brezgunov, V. N., Zlobin, V. N., Udaltsov, V. A. 1972, Astron. Journ. (USSR), 49, 279.

15. Brown, L. W., Alexander, J. K. 1971, Bull. Amer. Astr. Soc., 3, 469.

16. Burns, W. R., Clark, B. G. $\quad$ 1969, Astron. Astrophys., 2, 280.

17. Churchwell, E., Felli, M., Mezger, P. G. $\quad$ 1969, Astrophys. Letters, 4, 33. 
18. Churchwell, E., Felli, M. 1970, Astron. J., 75, 69.

19. Clark, R. R., Smith, F. G. 1969 , Nature, 221, 724.

20. Clark, T. A., Brown, L. W., Alexander, J. K. 1970, Nature, 228, 847.

21. Davies, J. G., Large, M. I., Pickwick, A. C. 1970, Nature, 227, 1123.

22. Davies, J. G., Large, M. I. 1970, Mon. Not. R. astr. Soc, 149, 301.

23. Davies, R. D. 1969 , Nature, 223, 355.

24. Dickel, H. R., Habing, H., Israel, F. P., Smith, L. F., Wendker, H. J. 1973, New Optical and Radio Data on NGC 6888, in preparation.

25. Dickel, J. R., McKinley, R. R. 1969, Astrophys. J., 155, 1.

26. Dickel, J. R. 1969, Astrophys. Letters, 4, 109.

27. Dickel, J. R., Milne, D. K. 1971, Physical Sciences, 231, 19.

28. Dickel, J. R., Milne, D. K., Ables, J. G., Kerr, A. R., Hermann, B. R. $\quad$ 1971, BAAS, 3, 451.

29. Dickel, J. R. $\quad 1971$, Pub. of the Astron. Society of the Pac., 83, 493.

30. Dickel, J. R. 1972, 'High Resolution Observations of Supernova Remnants at $80 \mathrm{MHz}$ ', in preparation.

31. Downes, D. 1970, Astron. J. 76, 305.

32. Downes, D., Maxwell, A., Rinehart, R. $\quad$ 1970, Astrophys. J., 161, L123.

33. Downs, G. S., Thompson, A. R. $\quad$ 1972, Astron J., 77, 120.

34. Drake, F. D. $\quad 1970$, Pub. Astron. Soc. Pac., 82, 395.

35. Drake, F. D. 1971, The Crab Nebula, ed. R. D. Davies and F. G. Smith, 73.

36. Drake, F. D. $\quad$ 1972, Cosmic Plasma Physics, ed. K. Schindler, 225.

37. Drake, F. D. 1972, Proc. of Conf. on Pulsating Radiosources and High Energy Activity in Supernova Remnants, Acad. dei Lincei, 7.

38. Dworetsky, M. M., Epstein, E. E., Fogarty, W. G., Montgomery, J. W. $\quad$ 1969, Astrophys. J., 158, L183.

39. Ekers, R., Lequeux, J., Moffet, A., Seielstad, G. $\quad$ 1969, Astrophys J., 156, L21.

40. Ekers, R. D., Lynden-Bell, D. 1971, Astrophys Letters, 9. 189.

41. Encrenaz, P. J., Penzias, A. A., Wilson, P. W. 1970 , Astrophys. J., 160, 1185.

42. Encrenaz, P., Foy, F., Guélin, M. 1970, C. R. Acad. Sci., 270, B, 1585.

43. Encrenaz, P., Falgarone, E., Franquelin, O., Guélin, M. 1971, Acad. Sci., 273. 686.

44. Ephanov, V. A., Kislyakov, A. J., Moiseev, I. G., Naumov, A. I. 1972, Astron. Journ. (USSR), 48, 435 .

45. Erickson, W. C., Kuiper, T. B. H., Clark, T. A., Knowles, S. H., Broderick, J. J. $\quad$ 1972, Astrophys. $J ., 177,101$.

46. Erkes, J. W., Dickel, J. R. 1969 , Astron. J. ,74, 840.

47. Ewing, M. S., Batchelor, R. A., Friefeld, R. D., Price, R. M., Staelin, D. H. $\quad$ 1970, Astrophys. J., 162, L169.

48. Felli, M., Churchwell, E. $\quad 1970$, Astrophys. J., 160, 43.

49. Goldstein, M. L., Ramaty, R., Fisk, L. A. 1970, Phys. Rev. Letters, 24, 1193.

50. Goldstein, Jr., S. J., Meisel, D. D. $\quad 1969$, Science, 163, 810.

51. Goldstein, Jr., S. J., James, J. T. $\quad$ 1969, Astrophys. J., 158, L179.

52. Goldstein, Jr., S. J., Meisel, D. D. $\quad$ 1969, Nature, 224, 349.

53. Galt, J. A., Lyne, A. G. $\quad 1972$, Mon. Not. R. Astr. Soc., 158, 281.

54. Gordon, K. J., Gordon, C. P. $\quad$ 1970, Astrophys. Letters, 5, 153.

55. Gordon, M. A., Williams, T. B. $\quad$ 1971, Astron. Astrophys., 12, 120.

56. Graham, D. A., Lyne, A. G., Smith, F. G. $\quad$ 1970, Nature, 225, 526.

57. Graham, D. A. 1971, Nature, 229, 326.

58. Hakura, Y., Nishizaki, R., Tao, K. 1969, J. Radio Res. Labs., Japan, 16, 215.

59. Herrero, V., Hjellming, R. M., Wade, C. M. 1971, Astrophys. J., 166, L19.

60. Hirabayashi, H., Yokoi, H., Morimoto, M. 1972, Nature, 237, 54.

61. Hirabayashi, H., Takahashi, T. 1972, Publ. Astr. Soc. Japan, 24, 231.

62. Hjellming, R. M., Wade, C. M. 1970, Astrophys. J., 162, L1.

63. Hjellming, R. M., Wade, C. M. 1971, Astrophys. J., 168, L87.

64. Hjellming, R. M., Wade, C. M. 1971, Astrophys. J., 168, L21.

65. Hjellming, R. M., Wade, C. M. 1971, Astrophys. J. ,164, L1.

66. Hjellming, R. M., Wade, C. M. 1971, Science, 173. 1087.

67. Hjellming, R. M., Wade, C. M., Hughes, V. A., Woodsworth, A. 1971, Nature, 234, 138.

68. Hobbs, R. W., Corbett, H. H., Santini, N. J. $\quad$ 1969, Astrophys. J., 155, L87. 
69. Hobbs, R. W., Corbett, H. H., Santini, N. J. $\quad$ 1969, Astron. J., 74, 824.

70. Hobbs, R. W., Modali, S. B., Maran, S. P. 1971, Astrophys. J., 165, L87.

71. Hobbs, R. W., Marionni, P. 1971, Astrophys. J., 167, 85.

72. Hollinger, J. P., Hevey, R. H., Knowles, S. H., Sadeh, D., Youmans, A. B. 1970 , Astrophys. J. Letters, 162, L105.

73. Hobbs, R. W., Johnston, K. J. 1971, Astrophys. J. 163, 299.

74. Hobbs, R. W., Waak, J. A. 1971, Publ. Astron. Soc. Pacific, 83, 166.

75. Hobbs, R. W., Waak, J. A. 1972, Astron. J., 77, 342.

76. Huguenin, G. R., Taylor, J. H. 1969, Astrophys. Letters, 3, 107.

77. Huguenin, G. R., Taylor, J. H., Jura, M. 1969, Astrophys. Letters, 4, 71.

78. Huguenin, G. R., Taylor, J. H., Troland, T. H. 1970 , Astrophys. J., $162,727$.

79. Huguenin, C. R., Manchester, R. N., Taylor, J. H. $\quad 1971$, Astrophys. J., $169,97$.

80. Huguenin, G. R., Taylor, J. H., Hjellming, R. M., Wade, C. M. 1971 , Nature-Phys. Sci., 234, 50.

81. Hunt, G. C. 1969, Nature, 224, 1005.

82. Hunt, G. C. $\quad$ 1971, Mon. Not. R. Astr. Soc., 153, 119.

83. Ilovaisky, S., Lequeux, J. $\quad 1972$, Astron. Astrophys, 18, 185.

84. Isobe, S., Kawaziri, N., Ojima, T., Kawano, N., Kurihara, H.

1972, Tokyo Astron. Bull., 218, 2549.

85. Johnson, H. M. 1971, Astrophys. J., 167, 491.

86. Johnson, H. M., Rubin, R. H. $\quad 1971$, Astrophys. J., 163, 151.

87. Johnston, K. J., Hobbs, R. W. $\quad$ 1969, Astrophys. J., 158, 145.

88. Kaftan-Kassim, M. A. 1969 , Astrophys. J., 155, 469.

89. Kaifu, N., Akabane, K., Morimoto, M. 1973, Publ. Astr. Soc. Japan, 25, No. 1, in press.

90. Kazes, I. 1971, Astron. Astrophys., 15, 460.

91. Kazes, I., Aubry, D. 1973 , Astron. Astrophys., in press.

92. Kellermann, K. I., Pauliny-Toth, I. I. K. 1971, Astrophys. J., 166, L17.

93. Kerr, F. J., Beard, M. 1969, Australian J. Phys., 22121.

94. Kerr, F. J. 1971, Structure and Evolution of the Galaxy, 135-144, ed. L. N. Mavridis, Reidel.

95. Kerr, F. J., Sinclair, M. W. 1971, Australian J. Phys., 24, 769.

96. Kostenko, V. I., Matvejenko, L. I. 1970, Radiofizika, 13, 1433.

97. Kr'ymin, V. V. 1971, Radiofizika, 14, 199.

98. Kundu, M. R. 1969, Astrophys. J., 158, L103.

99. Kundu, M. R., Velusamy, T. 1969, Astrophys. J., 155, 807.

100. Kundu, M. R. 1970, Astrophys. J., 162, 17.

101. Kundu, M. R., Velusamy, T. 1971, Astrophys. J., 163, 231.

102. Kundu, M. R., Velusamy, T. 1971, Nature-Phys. Sci., 234, 54.

103. Kundu, M. R. 1971 , Astrophys. J., 165, L55.

104. Kurilchik, V. N. 1971, Astron. Circ., 629, 2.

105. Lang, K. R., Rickett, B. J. $\quad 1970$, Nature, 225, 528.

106. Larionov, M. G. $\quad 1971$, Astron. Circ., 630, 1.

107. Lipovka, N. M. 1971 , Astron. Journ. 48, 260 (USSR).

108. Lovell, A. C. B. $\quad 1971$, Quart. J. Roy. Astron. Soc. 12, 98.

109. Lyne, A. G. $\quad 1971$, Mon. Not. R. Astr. Soc., 153, 27 P.

110. Lyne, A. G., Smith, F. G., Graham, D. A. 1971, Mon. Not. R. Astr. Soc., 153, 337.

111. Lyne, A. G., Smith, F. G. 1972, Mon. Not. R. Astr. Soc., 157, 15P.

112. Manchester, R. N. 1970, Nature, 225, 1124.

113. Manchester, R. N. 1971, laU Symposium, 46, 209.

114. Manchester, R. N. 1970, Nature, 228, 264.

115. Manchester, R. N. $\quad 1971$, IAU Symposium, 46, 118.

116. Manchester, R. N. $\quad$ 1971, Astrophys. J. Suppl. Ser., 23, 283.

117. Manchester, R. N. 1971, Astrophys. J., 163, L61.

118. Manchester, R. N. 1971, Astrophys. J., 167, L101.

119. Manchester, R. N. 1971, Nature-Phys. Sci., 231, 189.

120. Manchester, R. N., Tademaru, E. $\quad$ 1971, Nature-Phys. Sci., 232, 164.

121. Matvejenko, L. I., Conklin, E. K. 1972 , Astron. Journ. (USSR), 49, 895.

122. McCutcheon, W. H., Shuter, W. L. H. 1971, Astrophys. Letters, 9, 201.

123. Mezger, P. G. 1970, IAU Symposium, 38, 107.

124. Mezger, P. G. $\quad 1970$, IAU Symposium, 39, 336.

125. Mezger, P. G. $\quad$ 1970, Mem. Soc. Roy. Sci. Liège, 19, 325. 
126. Miley, G. K., Turner, B. E., Balick, B., Heiles, C. $\quad$ 1970, Astrophys. J., 160, L119.

127. Miley, G. K., Webster, Jr., W. J., Fullmer, J. W. 1970, Astrophys. Letters, 6, 17.

128. Montgomery, J. W., Epstein, E. E., Oliver, J. P., Dworetsky, M. M., Fogarty, W. G. 1971, Astrophys J., 167, 77.

129. O'Dell, C. R., Terzian, Y. $\quad$ 1970, Astrophys J., 160, 915.

130. Pfleiderer, J. 1970, Nature, 225, 437.

131. Pfleiderer, J. 1971, Astron. Astrophys., 13, 496.

132. Price, R. M. $\quad 1969$, Australian J. of Phys., 22, 641.

133. Price, R. M. 1970, Australian J. of Phys., 23, 227.

134. Price, R. M. 1970, Astron. J., 75, 144.

135. Reifenstein III, E. C., Brundage, W. D., Staelin, D. H. $\quad$ 1969, Phys. Rev. Letters, 22, 311.

136. Reifenstein III, E. C., Conklin, E. K., Staelin, D. H. $\quad$ 1969, Astrophys. J., 156, L125.

137. Rickett, B. J. 1970, Mon. Not. R. Astr. Soc., 150, 67.

138. Rubin, R. H. $\quad 1969$, Astrophys. J. 157, 1461.

139. Rubin, R. H., Turner, B. E. $\quad 1971$, Astrophys. J., 165, 471.

140. Schonhardt, R. E., Large, M. I., Lyne, A. G., Graham, D. A., Conway, R. G., Smith, F. G. 1971 , IAU Symposium, 46, D. Reidel, Co.

141. Schraml, J., Mezger, P. G. $\quad$ 1969, Astrophys. J., 156, 269.

142. Shitov, Yu. P. 1972, Astron. Journ. (USSR), 49, 470.

143. Shuter, W. L. H., McCutcheon, W. H. 1970, Nature, 227, 1331.

144. Shvartsman, V. F. $\quad 1971$, Astron. Journ. (USSR), 48, 438; 1972, Soviet Astr., 15, 342.

145. Smith, F. G. $\quad 1969$, Nature, 223, 934.

146. Smith, F. G. $\quad 1970$, Mon. Not. R. astr. Soc., 149, 1.

147. Smith, F. G. $\quad 1970$, Nature, 228, 913.

148. Smith, F. G. 1971, Nature, Phys. Sci., 231, 191.

149. Smith, F. G. $\quad 1971$, Mon. Not. R. astr. Soc., 154, 5P.

150. Smith, F. G. 1971, Nature, Phys. Sci., 232, 165.

151. Smith, F. G. 1972, Proc. of Meeting on Pulsars and High Energy Activity in Supernovae Remnants. Rome.

152. Smith, F. G. $\quad$ 1972, Reports on Progress in Physics, 4, 399.

153. Soboleva, N. S. $\quad 1973$, Izvestia Specialnoj Astrof. Observ., 5, in press.

154. Soboleva, N. S., et al, 1973 , Astron. Journ., USSR, 50, in press.

155. Staelin, D. H., Reifenstein III, E. C. $\quad$ 1969, Astrophys. J., 156, L121.

156. Staelin, D. H., Ewing, M. S., Price, R. M., Sutton, J. M. $\quad$ 1970, Astrophys. J., 160, L7.

157. Staelin, D. H., Sutton, J. M. 1970, Nature, 226, 69.

158. Sutton, J. M., Staelin, D. H., Price, R. M., Weimer, R. $\quad$ 1970, Astrophys. J., 159, L89.

159. Sutton, J. M., Staelin, D. H., Price, R. M. 1971, IAU Symposium, 46, 97.

160. Tademaru, E. $\quad 1971$, Astrophys. Space Sci., 12, 193.

161. Taylor, J. H., Huguenin, G. R. 1971, Astrophys. J., 167, 273.

162. Taylor, J. H., Huguenin, G. R., Hirsch, R. M., Manchester, R. N. $\quad$ 1971, Astrophys. Letters, 9, 204.

163. Terzian, Y. 1969, Astrophys. Letters, 3, 87.

164. Terzian, Y. $\quad 1970, A . J ., 75,1155$.

165. Terzian, Y., Parrish, A. $\quad$ 1970, Astrophys Letters, 5, 261.

166. Terzian, Y. $\quad 1971$, Ap. J. 166, 559.

167. Terzian, Y. 1972, The Physics of Pulsars, ed. A. M. Lenchek, Gordon and Breach, 85.

168. Terzian, Y. 1972, Earth and Extraterrestrial Sciences, 2, 63.

169. Terzian, Y., Pankonin, V. $\quad 1972$, A. J., 77, 115.

170. Terzian, Y., Sanders, D. $1972, A . J ., 77,350$.

171. Troitskij, V. S. $\quad 1971$, Astron. Journ. (USSR), 48, 645.

172. Tsarevskij, G. S. 1972 , Astrophys Letters $10,71$.

173. Vitkevich, V. V., Malofeev, V. M., Shitov, Yu. P. 1971, Astron. Journ. (USSR), 48, 1333.

174. Vitkevitch, V. V., et al. 1972, Ap. J., 9, 209.

175. Wade, C. M., Hjellming, R. M. 1971, Astrophys. J., 163, L105.

176. Wade, C. M., Hjellming, R. M. 1971, Astrophys. J., 170, 523.

177. Wade, C. M., Hjellming, R. M. 1971, Astrophys. J., 163, L65.

178. Webb, H. D., Dickel, J. R. 1971, Astron. Journal, 76, 3.

179. Webster, Jr., W. J., Altenhoff, W. J. $\quad 1970$, Astrophys. Letters, 5, 233.

180. Webster, Jr., W. J., Altenhoff, W. J. $\quad 1970$, Astron. J., 75, 896. 
181. Webster, Jr., W. J., Wink, J. E., Altenhoff, W. J. 1970, Astrophys. Letters, 7, 47.

182. Webster, W. J., Altenhoff, W. J., Wink, J. E. $\quad 1971$, Astron. J., 76, 677.

183. Weiler, K. W., Seielstad, G. A. $\quad 1971$, Astrophys. J., 163, 455.

184. Weiler, K. W., Seielstad, G. A. $\quad 1972$, Astron. Astrophys, $21,393$.

185. Wendker, H. J. 1970, Astron. Astrophys., 4, 378.

186. Wendker, H. J., Dickel, J. R., Yang, K. S., and Staff (Vermilion River Observatory, University of Illinois) 1970, Astron. J., 75, 148.

187. Wendker, H. J. 1971, Astron. Astrophys., 13, 65.

188. Willis, A. G., Dickel, J. R. $\quad$ 1971, Astrophys. Letters, 8, 203.

189. Wilson, T. L., Altenhoff, W. 1970, Astrophys. Letters, $5,47$.

190. Wilson, T. L. 1970, IAU Symposium, 38, 140.

191. Wilson, W. J. 1971, Astrophys. J., 166, L13.

192. Yukimuk, A. K. 1971, Astrofizika (USSR), 7, 611.

193. Zabolotnyj, I. V. F. 1971 , Astron. Circ., 635, 1.

194. Zheleznyakov, V. V. 1971, Astrophys. Space Sci., 13, 74.

195. Backer, D. C. $\quad 1970$, Nature, 228, 42.

196. Backer, D. C. $\quad 1970$, Nature, 228, 752.

197. Backer, D. C. $\quad 1973$, Astrophys J., May issue.

\section{LINE RADIATION FROM THE GALAXY}

Ben Zuckerman

The goal of this section is to provide a complete bibliography of papers published in the past three years. A short list of various reviews and symposia is also included. The following discussion is an attempt to indicate what topic each reference is about. For a fuller discussion of the content of these papers, the reader is referred to the relevant reports to Commission 34 in the present volume and especially to the reviews listed below.

\section{21-Centimeter hydrogen line}

Hydrogen line absorption interferometry in the Northern (165) and Southern $(139,270,271,272$, 273) hemispheres has yielded important new information relating to the two-component models of $\mathrm{H}$ I regions. Surveys of various regions with single antennas have also been carried out in the Northern $(7,30,31,74,75,76,217,265,279,320,321,322,325,349,368,369,374,386,393)$ and Southern $(139,154,187,188,190,271,272,273)$ hemispheres. From these, various pictures of galactic structure $(32,33,34,95,167,197,227,228,289,290,291,292)$ are obtained. Specific sources have been examined $(18,19,45,57,92,109,110,123,124,125,138,162,166,170,184,185,193,202$, $232,277,302,306,347,348,371,377,379,380,391,416)$. The high velocity clouds $(62,87,168$, $192,195,205,206,231,242,276,372,381,384,387,388$ ) and high velocity gas near the galactic center $(203,204,234,307,308,309)$ have received special attention. 21-centimeter emission and/or absorption has been used to obtain pulsar distances $(86,126,127,143,144,225,304)$ and to study cold clouds and the abundance ratio of dust and gas $(221,268,279,293,303,305,324,339,373$, $385,392)$ and magnetic fields through the Zeeman effect $(24,370,375,376,382)$. Papers have been written on determination of the astronomical unit (244), 21-cm optical depths $(107,152)$, the total mass of atomic hydrogen gas in the galaxy (122), and lunar occultation in the $21-\mathrm{cm}$ line (201).

\section{Recombination lines}

The so-called 'diffuse' interstellar medium has recently been the object of considerable observational $(66,131,133,140,171)$ and theoretical $(40,130)$ work. Hydrogen lines from discrete $\mathrm{H}$ il regions in the Northern $(51,52,54,61,77,128,129,132,135,233,235,250,251,255,256,274,280,282,294$, 297, 298, 326, 337, 404, 405, 406) and Southern $(35,69,99,100,237,238,240,241,407)$ hemispheres have been investigated. Theoretical analysis of recombination lines from predominantly ionized $(4,5,20,21,22,23,83,117,156,157,158,159,160,253,263,318)$ and from predominantly 